\title{
Microbial degradation of chitin materials by Trichoderma virens UKM1
}

\begin{abstract}
The current increase in amount of seafood wastes produced by the shrimp industry has lead to the finding of new methods for shrimp waste disposal or waste reused. For this respect, chitinase-producing fungi have been extensively studied as biocontrol agents. Locally isolated Trichoderma viren UKM1 was used in this study. From preliminary study, commercialized Trichoderma Minimal Medium (TMM) was selected for the degradation study. The substrates used were colloidal chitin as control substrate, sun dried ground and unground shrimp shells. Scanning Electron Microscopy (SEM) studies showed penetration of fungus mycelium into the colloidal chitin as compare to sun dried ground and unground. This observation suggested that colloidal chitin was the best carbon source for modeling the degradation of chitin materials. Stereo microscope studies suggested that the fungus removed (degraded) the chitinous materials layer by layer as indicated by the significant reduction in shell thickness. Shrimp shells were further evaluated for end products in the crude medium using High Performance Liquid Chromatography (HPLC). A simple, rapid, selective and specific HPLC method was developed to quantify glucosamine indirectly using the value of total $\mathrm{N}$-acetyl-glucosamine (NAG) produced which the production of chitooligomer was used as marker. Results showed that the Trichoderma virens UKM1 secretes a significant amount of exochitinase compared to endochitinase by the identification of monomeric N-acetylglucosamine (NAG) from the chitinous substrate. The highest specific enzyme activity obtained using colloidal chitin was $14.59 \mathrm{U}$ mg-1. Percentage of residual chitooligomer in impure chitinases samples was $86 \%$.
\end{abstract}

Keyword: Degradation; Trichoderma virens UKM1; Chitoologomer; Chitinase; HPLC 